\title{
Aspirin versus anticoagulation in cervical artery dissection (TREAT-CAD): an open-label, randomised, non-inferiority trial
}

Engelter, Stefan T ; Traenka, Christopher ; Gensicke, Henrik ; Schaedelin, Sabine A ; Luft, Andreas R ; et al ; Steiner, Levke ; Hamann, Janne ; Stippich, Christoph ; Wegener, Susanne ; Psychogios, Marios-Nikos

\begin{abstract}
Background Cervical artery dissection is a major cause of stroke in young people (aged $<50$ years). Historically, clinicians have preferred using oral anticoagulation with vitamin $\mathrm{K}$ antagonists for patients with cervical artery dissection, although some current guidelines - based on available evidence from mostly observational studies - suggest using aspirin. If proven to be non-inferior to vitamin $\mathrm{K}$ antagonists, aspirin might be preferable, due to its ease of use and lower cost. We aimed to test the noninferiority of aspirin to vitamin $\mathrm{K}$ antagonists in patients with cervical artery dissection. Methods We did a multicentre, randomised, open-label, non-inferiority trial in ten stroke centres across Switzerland, Germany, and Denmark. We randomly assigned (1:1) patients aged older than 18 years who had symptomatic, MRI-verified, cervical artery dissection within 2 weeks before enrolment, to receive either aspirin $300 \mathrm{mg}$ once daily or a vitamin $\mathrm{K}$ antagonist (phenprocoumon, acenocoumarol, or warfarin; target international normalised ratio [INR] $2 \cdot 0-3 \cdot 0$ ) for 90 days. Randomisation was computer-generated using an interactive web response system, with stratification according to participating site. Independent imaging core laboratory adjudicators were masked to treatment allocation, but investigators, patients, and clinical event adjudicators were aware of treatment allocation. The primary endpoint was a composite of clinical outcomes (stroke, major haemorrhage, or death) and MRI outcomes (new ischaemic or haemorrhagic brain lesions) in the per-protocol population, assessed at 14 days (clinical and MRI outcomes) and 90 days (clinical outcomes only) after commencing treatment. Non-inferiority of aspirin would be shown if the upper limit of the two-sided $95 \%$ CI of the absolute risk difference between groups was less than $12 \%$ (non-inferiority margin). This trial is registered with ClinicalTrials.gov, NCT02046460. Findings Between Sept 11, 2013, and Dec 21, 2018, we enrolled 194 patients; 100 (52\%) were assigned to the aspirin group and 94 (48\%) were assigned to the vitamin K antagonist group. The per-protocol population included 173 patients; 91 (53\%) in the aspirin group and $82(47 \%)$ in the vitamin $\mathrm{K}$ antagonist group. The primary endpoint occurred in 21 (23\%) of 91 patients in the aspirin group and in $12(15 \%)$ of 82 patients in the vitamin $\mathrm{K}$ antagonist group (absolute difference $8 \%$ [95\% CI -4 to 21], non-inferiority $\mathrm{p}=0 \cdot 55$ ). Thus, non-inferiority of aspirin was not shown. Seven patients $(8 \%)$ in the aspirin group and none in the vitamin $\mathrm{K}$ antagonist group had ischaemic strokes. One patient (1\%) in the vitamin K antagonist group and none in the aspirin group had major extracranial haemorrhage. There were no deaths. Subclinical MRI outcomes were recorded in 14 patients (15\%) in the aspirin group and in 11 patients $(13 \%)$ in the vitamin $\mathrm{K}$ antagonist group. There were 19 adverse events in the aspirin group, and 26 in the vitamin $\mathrm{K}$ antagonist group. Interpretation - Our findings did not show that aspirin was non-inferior to vitamin $\mathrm{K}$ antagonists in the treatment of cervical artery dissection.
\end{abstract}

DOI: https://doi.org/10.1016/s1474-4422(21)00044-2 
ZORA URL: https://doi.org/10.5167/uzh-203241

Journal Article

Accepted Version

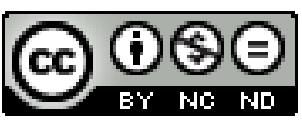

The following work is licensed under a Creative Commons: Attribution-NonCommercial-NoDerivatives 4.0 International (CC BY-NC-ND 4.0) License.

Originally published at:

Engelter, Stefan T; Traenka, Christopher; Gensicke, Henrik; Schaedelin, Sabine A; Luft, Andreas R; et al; Steiner, Levke; Hamann, Janne; Stippich, Christoph; Wegener, Susanne; Psychogios, MariosNikos (2021). Aspirin versus anticoagulation in cervical artery dissection (TREAT-CAD): an open-label, randomised, non-inferiority trial. Lancet Neurology, 20(5):341-350.

DOI: https://doi.org/10.1016/s1474-4422(21)00044-2 
Aspirin versus anticoagulation in cervical artery dissection: an open label, randomised, noninferiority trial (TREAT-CAD)

\section{Authors:}

Stefan $\mathrm{T}$ Engelter, $\mathrm{MD}^{1,2^{*} \dagger}$, Christopher Traenka, $\mathrm{MD}^{1,2^{*}}$, Henrik Gensicke, $\mathrm{MD}^{1,2}$, Sabine Schaedelin, $\mathrm{MSc}^{3}$, Andreas R. Luft, $\mathrm{MD}^{4,5 \dagger}$, Barbara Goeggel Simonetti, MD ${ }^{6,7}$, Urs Fischer, $\mathrm{MD}^{6 \dagger}$, Patrik Michel, $\mathrm{MD}^{8 \dagger}$, Gaia Sirimarco, $\mathrm{MD}^{8}$, Georg Kägi, $\mathrm{MD}^{9}$, Jochen Vehoff, $\mathrm{MD}^{9}$, Krassen Nedeltchev, $\mathrm{MD}^{10 \dagger}$, Timo Kahles, $\mathrm{MD}^{10}$, Lars Kellert, $\mathrm{MD}^{11,12 \dagger}$, Sverre Rosenbaum, $\mathrm{MD}^{13}$, Regina von Rennenberg, $\mathrm{MD}^{14}$, Roman Sztajzel, $\mathrm{MD}^{15 \dagger}$, Stephen L Leib, MD ${ }^{16 \dagger}$, Simon Jung, MD ${ }^{6 \dagger}$, Jan Gralla, $\mathrm{MD}^{17 \dagger}$, Nicole Bruni, $\mathrm{PhD}^{3}$, David Seiffge $\mathrm{MD}^{1,6}$, Katharina Feil, $\mathrm{MD}^{11}$, Alexandros A Polymeris, $\mathrm{MD}^{1}$, Levke Steiner, $\mathrm{MD}^{4}$, Janne Hamann, $\mathrm{MD}^{4}$, Leo H Bonati, $\mathrm{MD}^{1 \dagger}$, Alex Brehm, $\mathrm{MD}^{18}$, Gian Marco De Marchis, MD, MSc ${ }^{1,6}$, Nils Peters, $\mathrm{MD}^{1,2 \dagger}$, Christoph Stippich, $\mathrm{MD}^{19 \dagger}$, Christian $\mathrm{H}$ Nolte, $\mathrm{MD}^{14 \dagger}$, Hanne Christensen, $\mathrm{MD}^{13 \dagger}$, Susanne Wegener, $\mathrm{MD}^{4 \dagger}$, Marios-Nikos Psychogios, $\mathrm{MD}^{18 \dagger}$, Marcel Arnold, $\mathrm{MD}^{6 * \dagger}$, Philippe Lyrer, $\mathrm{MD}^{1 * \dagger}$, for the TREAT-CAD investigators

*Dr Engelter and Dr Traenka contributed equally.

*Dr Arnold and Dr Lyrer contributed equally.

\section{Authors are full professors.}

A complete list of TREAT-CAD investigators, members of the Steering Committee, the Data Safety Monitoring Board, the Clinical Event Adjudication Committee, and the Independent Imaging Core Lab Adjudicators is provided in the supplementary appendix. 
Engelter, Traenka et al. TREAT-CAD main results - 2 -

\section{Affiliations:}

${ }^{1}$ Department of Neurology and Stroke Center, University Hospital Basel and University of Basel, Switzerland

${ }^{2}$ Neurology and Neurorehabilitation, University Department of Geriatric Medicine FELIX PLATTER, University of Basel, Basel Switzerland

${ }^{3}$ Department of Clinical Research, Clinical Trial Unit, University Hospital Basel and University of Basel, Switzerland

${ }^{4}$ Division of Vascular Neurology and Neurorehabilitation, Department of Neurology, University Hospital of Zurich, and University of Zurich, Switzerland

${ }^{5}$ Cereneo, Center for Neurology and Rehabilitation, Vitznau, Switzerland

${ }^{6}$ Department of Neurology, University Hospital Bern, University of Bern Switzerland

${ }^{7}$ Department of Neuropediatrics, Institute of Pediatrics of Southern Switzerland, San Giovanni Hospital, 6500

Bellinzona

${ }^{8}$ Stroke Center and Neurology Service, Department of Clinical Neurosciences, Centre Hospitalier Universitaire Vaudois and University of Lausanne, Switzerland

${ }^{9}$ Department of Neurology and Stroke Center, Cantonal Hospital St.Gallen, St.Gallen, Switzerland

${ }^{10}$ Department of Neurology and Stroke Center, Cantonal Hospital Aarau, Aarau, Switzerland

${ }^{11}$ Department of Neurology, Ludwig Maximilian University (LMU), Munich, Germany

${ }^{12}$ Institute for Stroke and Dementia Research (ISD), University Hospital, LMU Munich, Munich, Germany

${ }^{13}$ Department of Neurology, Bispebjerg Hospital \& University of Copenhagen, Copenhagen, Denmark

${ }^{14}$ Klinik und Hochschulambulanz für Neurologie, Charité-Universitätsmedizin Berlin, corporate member of Freie Universität Berlin, Humboldt-Universität zu Berlin, Berlin Institute of Health, Germany and Center for Stroke Research Berlin (CSB), Charité-Universitätsmedizin Berlin, Germany

${ }^{15}$ Department of Neurology and Stroke center, University Hospital Geneva and Medical School, Geneva, Switzerland

${ }^{16}$ Institute for Infectious Diseases, University of Bern, Switzerland

${ }^{17}$ Institute of Diagnostic and Interventional Neuroradiology, University Hospital Bern, University of Bern, Switzerland

${ }^{18}$ Department of Neuroradiology, Clinic of Radiology and Nuclear Medicine, University Hospital Basel, Basel, Switzerland

${ }^{19}$ Department of Neuroradiology, University Hospital Zurich, Switzerland 


\title{
Corresponding author:
}

Prof. Dr. med Stefan Engelter

Department of Neurology and Stroke Center, University Hospital and University of Basel, Petersgraben 4, CH - 4031 Basel, Switzerland

Email: stefan.engelter@usb.ch

\section{And:}

Neurology and Neurorehabilitation, University Department of Geriatric Medicine FELIX PLATTER, University of Basel, Department of Clinical Research

Burgfelderstrasse 101, CH-4002 Basel, Switzerland

Phone: 0041 - 61326 4105, Fax: 0041 - 612655544

\section{Word / character count:}

\author{
Abstract: 417 \\ Research in context: 523 \\ Main manuscript: 4555
}

Title (character): 109

\section{References: $\mathbf{n}=\mathbf{3 7}$}

Tables: $\mathbf{n}=\mathbf{3}$

Figures: $\mathbf{n = 2}$ 


\section{Sources of funding for TREAT-CAD:}

Swiss National Science Foundation

Swiss Heart Foundation

Stroke Funds Basel

University Hospital Basel

University of Basel

Academic Society Basel [Freiwillige Akademische Gesellschaft Basel, FAG]

Search terms: Cervical Artery Dissection, Stroke, Prevention, Randomised Clinical Trial, Antiplatelets, Anticoagulants

\section{$\underline{\text { Tables and Figures }}$}

Table 1: Characteristics of patients at baseline

Table 2: Primary and Secondary Endpoints

Table 3: Adverse Events

Figure 1: Study flow-chart

Figure 2: Main analyses and sensitivity analyses

\section{Supplementary Appendix}

Steering Committee members; Clinical Event Adjudication Committee members; Independent Imaging Core Lab Adjudicators; Data Safety Monitoring Board members; Statistical analysis team; Study coordination and independent monitoring; Participating centers, numbers of recruited patient, investigators; Supplementary methods; Supplementary results; Study protocol; Statistical analysis plan. 


\section{Author contributions:}

STE, $C T, M A, P L$ study design, selecting participating centers, acquisition of funding, grant and ethical submission, protocol development, data collection and interpretation, drafting the manuscript.

\section{STE, CT have verified all underlying data}

$H G, L H B$ study design, acquisition of funding, protocol development, data collection and interpretation, critical review of the manuscript.

SAS study design, statistical analysis, data interpretation, drafting the manuscript.

$N B$ study design, trial operations, site monitoring, data management, critical review of the manuscript.

$A L, P M, G K, K N, T K, L K, S R, R S, C H N, H C, S W, C S$ study design, protocol development, data collection and interpretation, critical review of the manuscript.

$B G S, S L, S J, J G, U F, D S, K F, A P, L S, J H, G M D M, N P, G S, J V, R V R$ data collection, data interpretation, and critical review of the manuscript.

$A B, M N P$ imaging core lab adjudication, data interpretation, critical review of manuscript. All authors read and approved the final manuscript.

Conflicts of interest: The authors report no disclosures relevant to the manuscript. 


\section{Abstract}

Background: Cervical artery dissection $(\mathrm{CAD})$ is a major cause of stroke in young people $(<50$ years). Historically, clinicians preferred oral anticoagulation using Vitamin K Antagonists (VKAs), while some current guidelines - based on available evidence from mostly observational studies -, suggested aspirin. If proven non-inferior to VKA, aspirin might be preferable due to easier handling and lower cost. We therefore aimed to test the non-inferiority of aspirin to VKA in CAD patients. Methods: In a multicentre (10 stroke centres in Switzerland, Germany, Denmark), randomised, open-label, non-inferiority trial with blinded assessment of endpoints, we randomly allocated patients with symptomatic CAD verified by Magnetic Resonance Imaging (MRI) to receive either aspirin $300 \mathrm{mg}$ daily or VKA (target INR 2-3) for 90 days (ratio 1:1). Randomisation was computer-generated using an interactive web response system, with stratification according to participating site. Independent imaging core lab adjudicators were masked to the treatment allocation, but investigators, patients and clinical event adjudicators were aware of treatment allocation. Primary endpoint was a composite of clinical (stroke, major haemorrhage, death) and MRI-outcomes (new ischemic or haemorrhagic brain lesions) in the per protocol population, assessed at 14 (clinical and MRI-outcomes) and 90 days (clinical outcomes) after treatment onset. Non-inferiority of aspirin would be shown if the upper limit of the two-sided $95 \%$ confidence interval $(\mathrm{CI})$ of the absolute risk difference between groups was less than $12 \%$ (non-inferiority margin). The trial was registered with https://clinicaltrials.gov/ct2/show/NCT02046460.

Findings: Among 194 randomised patients (recruited and assigned to aspirin $n=100$, recruited and assigned to VKA n=94) enrolled between September 2013 and December 2018, the per protocol population included 173 patients (aspirin group $n=91$, VKA group $n=82$ ). The primary endpoint occurred in 21 of $91(23.1 \%)$ patients in the aspirin-group and in 12 of 82 (14.6\%) patients in the VKA-group (absolute difference $8.5 \%, 95 \%$-CI -4.3 to $21.2 \%$, p for non-inferiority=0.55). Thus, 
non-inferiority of aspirin was not shown. Seven patients (7.7\%) in the aspirin-group and none in the VKA-group had ischemic strokes. One patient (1.2\%) in the VKA-group and none in the aspirin-group had major extracranial haemorrhage. There were no deaths. Subclinical MRIoutcomes were recorded in $14(15.4 \%)$ patients in the aspirin-group and in $11(13.4 \%)$ patients in the VKA-group. Nineteen adverse events occurred in the aspirin group, and 26 in the VKA group. Interpretation: The evidence to consider aspirin as the standard of care in patients with CAD is weak. The fact that aspirin was not shown non-inferior to VKA does not mean that aspirin is worse and VKA is superior to aspirin.

Funding: Swiss National Science Foundation, Swiss Heart Foundation, Stroke Funds Basel, University Hospital Basel, University of Basel, Academic Society Basel, Switzerland 


\section{Research in context}

Evidence before this study: Cervical artery dissection (CAD) is a major cause of stroke in the young $(<50$ years $)$ and the appropriate antithrombotic treatment is unclear. We searched $P U B M E D$, EMBASE, The Cochrane library, and the clinicaltrials.gov registry (last access on August $27^{\text {th }}$ 2020) to identify randomised controlled trials (RCTs) and meta-analyses comparing antiplatelets versus anticoagulants in CAD patients (date range: from database inception until August $27^{\text {th }}$ 2020; no language restrictions). We used the following search terms: (dissection AND anticoagulants AND antiplatelets AND (carotid OR vertebral)) on PUBMED, The Cochrane Library and clinicaltrials.gov and ('dissection'/exp OR dissection) AND ('antithrombocytic agent'/exp OR 'antithrombocytic agent') AND ('carotid artery'/exp OR 'carotid artery' OR 'vertebral artery'/exp OR 'vertebral artery') on EMBASE.

We identified six meta-analyses - including one Cochrane Review - across observational data comparing antiplatelets to anticoagulants in CAD patients. In summary, they showed inconclusive results. There is one completed randomised controlled trial (RCT), the Cervical Artery Dissection in Stroke Study (CADISS). The CADISS investigators found that recurrent strokes occurred in only $2 \%$ of 197 patients. Recurrent strokes occurred - numerically - more often in the antiplatelet group (i.e., three out of 101 patients) than in the anticoagulation group (one out of 96) and the only major haemorrhage occurred in the anticoagulation group as well, indicating no clear difference between an antiplatelet and anticoagulant regimen. Furthermore, the lower than expected clinical event rate in CADISS indicated also that a RCT based purely on clinical endpoints is not feasible. The fact that in one out of five CADISS-patients, the diagnosis of CAD could not be confirmed in central imaging reading, suggested that the correct diagnosis of CAD is challenging in the acute phase. 
Most clinicians have traditionally favoured to use anticoagulants (mainly Vitamin-K-Antagonists, VKA) in CAD-patients, but since the publication of the CADISS results, this practice has often been abandoned, although this approach is not well supported by the available evidence.

Added value of this study: As prior evidence suggested that a RCT in CAD patients comparing antiplatelets to anticoagulants would be unfeasible, TREAT-CAD implemented a novel approach with the use of a composite primary endpoint, including clinical (stroke, major haemorrhage, death) and MRI outcomes (new ischemic or haemorrhagic brain lesions). This enabled to lower the trial sample size to a feasible magnitude and allowed the successful completion of the trial. TREATCAD was designed to test the non-inferiority of aspirin to VKA treatment in CAD patients. Noninferiority of aspirin was not shown. Likewise, non-inferiority of aspirin was not shown when analysing clinical and MRI outcomes separately, nor across all sensitivity analyses.

Implications of all the available evidence: The evidence to consider aspirin as the standard of care in CAD patients is weak. Currently it seems not justified to replace the traditional standard treatment - anticoagulation - by aspirin, although the superiority of anticoagulation is also still unproven. The importance of the type of presenting symptom might be addressed in an individual patient data meta-analysis across CADISS and TREAT-CAD. Direct oral anticoagulants have a more favourable benefit-risk ratio and are more conveniently applicable than VKAs. Thus, benefits and harms of direct oral anticoagulants in CAD should be tested in future trials. 
Engelter, Traenka et al. TREAT-CAD main results - 10 -

\section{INTRODUCTION}

Cervical Artery Dissection (CAD) is the leading cause of stroke in the young $(<50$ years $) .{ }^{1}$ The optimal type of antithrombotic treatment is unclear. ${ }^{2}$ Historically, many clinicians preferred oral anticoagulation with Vitamin K Antagonists (VKAs). ${ }^{3}$ This approach is not evidence based ${ }^{4}$ and guidelines have either expressed no preference ${ }^{5,6}$ or suggested antiplatelets. ${ }^{7}$ Observational studies $^{8-13}$ and one randomised-controlled trial $(\mathrm{RCT})^{14}$, the Cervical Artery Dissection in Stroke Study (CADISS), comparing antiplatelets to VKAs were inconclusive. Aspirin might be preferred because it is more convenient to use and less costly than VKAs. ${ }^{15}$

However, to support a preference for aspirin, it remains to be shown whether aspirin is non-inferior to anticoagulants in CAD. Based on estimates derived from the aforementioned studies, a conventional trial testing this hypothesis is not feasible if based purely on clinical endpoints, because a large sample size would be required. ${ }^{10,15}$ However, sufficient power in a RCT may be achieved by combining both clinical and MRI-outcomes. For this approach, the ICSS-MR substudy served as a role model, as the comparative analysis of MRI-outcomes in both treatment arms (i.e. stenting versus carotid endarterectomy) resulted in virtually the same results as those in the main study based on pure clinical outcomes - with less than $10 \%$ of patients. ${ }^{16}$ More recently, the MRIsub-studies of the AVERROES ${ }^{17}$ and the COMPASS ${ }^{18}$ RCTs found, that clinical and MRIoutcomes were consistent and thus their combined use has been advocated for. In line with this recommendation, the REDUCE trial implemented clinical and MRI-outcomes as co-primary outcome and demonstrated the usefulness of this combination in a stroke prevention RCT testing Patent Foramen Ovale closure. ${ }^{19}$

With this approach, we conducted the Biomarkers and antithrombotic TREATment in Cervical Artery Dissection trial (TREAT-CAD) to test whether aspirin is non-inferior to VKA in CAD patients regarding efficacy and safety. 


\section{METHODS}

\section{Study design}

The TREAT-CAD-trial (NCT02046460) was a Prospective, Randomised controlled, Openlabelled, multicentre, non-inferiority trial with $\boldsymbol{B}$ linded assessment of outcome $\boldsymbol{E}$ vents (PROBEdesign). The trial was performed in 10 stroke centers in Switzerland, Germany, and Denmark (supplementary appendix, page 3 ). The trial design and rationale have been described previously. ${ }^{20}$ TREAT-CAD was approved by ethics committees and regulatory authorities in Switzerland, Germany, and Denmark. Independent on-site and centralized monitoring was performed by the Clinical Trial Unit of the University Hospital Basel, Switzerland.

The trial was conducted in accordance with the trial protocol (full text available in the supplementary appendix, page 7). The trial was reported in accordance with the statistical analysis plan which was finalized and signed December $18^{\text {th }}, 2019$ and published (https://clinicaltrials.gov/ct2/show/NCT02046460) prior to database closure (January $14^{\text {th }}, 2020$ ) and data analysis.

The Sponsor-Investigator (STE) and the Scientific Medical Trial Manager (ChT) wrote the first draft of the manuscript. All authors vouch for the completeness and accuracy of the data and analyses, for the reporting of the trial results as well as adverse events and for the adherence of the trial conduct to the protocol.

\section{Participants}

Potential participants were informed about the trial and the option to participate in TREAT-CAD by local investigators (supplementary appendix, page 3-4) after their treating physicians had informed the patients about the diagnosis and the therapeutic options as part of the clinical routine Patients aged $>18$ years, presenting at the participating centers with acute ischemic (transient 
Engelter, Traenka et al. TREAT-CAD main results - 12 -

ischemic attack or ischemic stroke) or non-ischemic (i.e. "local signs") symptoms of CAD within two weeks prior to enrolment were eligible for participation. Prior to enrolment, the clinical diagnosis of CAD had to be confirmed by MRI-techniques according to accepted CAD diagnostic criteria $^{1}$ (see the supplementary appendix, page 4). In case of preceding intravenous thrombolysis and/or endovascular treatment, enrolment was allowed only after a $24 \mathrm{~h}$ latency period.

Exclusion criteria were: (i) MR-contraindications, (ii) contraindications to the use of aspirin or anticoagulation (VKA, heparin) as outlined in corresponding Summary of Product Characteristics in the countries of randomisation or according to the judgment of the treating physician, and (iii) pregnancy. ${ }^{20}$ Written informed consent by the patient, by next-of-kin or an independent physician if applicable was required before enrolment in the trial.

\section{Randomisation and masking}

Randomisation was computer generated by using an interactive web response system with stratification according to the participating sites. The randomisation sequence was generated by members of the Clinical Trial Unit Basel. Patients were randomly allocated to one of the treatment groups with a simple randomisation algorithm, in order to reach an equal distribution within each center. Patients were randomised to receive either aspirin or VKA (ratio 1:1). Independent Imaging Core Lab Adjudicators were masked to the allocated treatment, but investigators, patients and clinical event adjudicators were aware of treatment allocation. Study investigators (as listed in the supplementary appendix, pages 3-4) entitled in the study delegation logs of each center, recruited participants, performed the randomisation and accordingly assigned the participants to the respective treatment. These investigators also performed follow-up visits. 
Engelter, Traenka et al. TREAT-CAD main results - 13 -

\section{Procedures}

Aspirin 300 mg q.d. was administered orally [Aspirin ${ }^{\mathrm{TM}}$ ]. In dysphagic patients, administration of aspirin $250 \mathrm{mg}$ q.d. intravenously [Aspegic ${ }^{\mathrm{TM}}$ ] was allowed, until swallowing function had recovered. VKA meant the use of phenprocoumon [Marcumar $\left.{ }^{\mathrm{TM}}\right]$, or acenocoumarol [Sintrom ${ }^{\mathrm{TM}}$ ], or warfarin [Marevan ${ }^{\mathrm{TM}}$ ], according to local practices and using medication (aspirin and VKA) commercially available in the respective centres and countries. The target INR was 2.0 - 3.0. Bridging VKA-treatment with i.v. heparin or low molecular weight heparin until the target INR had been reached, was recommended. INR monitoring during the study treatment period was left to the discretion of the treating physician according to common practice in each center. The use of low-dose, prophylactic heparin for prevention of deep-vein thrombosis was allowed in the aspirin group. Treatment duration within the trial was 90 days or until occurrence of the primary endpoint. Patients were followed up at $14( \pm 10)$ days after treatment onset for a first clinical and MRI (3 Tesla) assessment including the following MRI-sequences: (i) Diffusion Weighted Imaging (DWI) including Apparent Diffusion Coefficient (ADC) maps to detect new acute ischemic brain lesions, (ii) paramagnetic sequences, i.e. $\mathrm{T} 2{ }^{*} \mathrm{w}$ gradient echo (GRE) or Susceptibility Weighted Images (SWI) to detect new haemorrhagic brain lesions (iii) contrast-enhanced magnetic resonance angiography (CE-MRA) with fat-suppression to improve delineation of the wall hematoma against the perfused vessel lumen.

At $90 \pm 30$ days patients were followed-up for a second clinical assessment. Follow-up visits were performed also in participants who had had primary endpoints prior to scheduled visits or in whom major protocol violations occurred. 
Engelter, Traenka et al. TREAT-CAD main results - 14 -

\section{Outcomes}

The primary endpoint was a composite of clinical (ischemic stroke, major extracranial or intracranial haemorrhage, death) and MRI-outcomes (new ischemic or haemorrhagic brain lesions), which were defined applying established criteria. ${ }^{21-23}$ Definitions of outcomes are given in the supplementary appendix (supplementary appendix, pages 4-5).

Clinical components of the primary endpoint were independently adjudicated by the Clinical Event Adjudication Committee, which was blinded to the allocated treatment. The MRI components of the primary endpoint were centrally adjudicated by an independent Imaging Core Lab which was blinded to the allocated treatment and the clinical outcome of participants. Confirmation of the CAD-diagnosis was done centrally in a consensus reading by two experienced investigators (STE, ChT). Secondary endpoints included - next to all components of the composite primary endpoint analysed separately - (i) any increase (compared to baseline) in volume of the vessel wall hematoma (as detected in the follow-up cervical MRI), (ii) functional outcome at 3 months as assessed with the modified Rankin Scale score, and (iii) any transient ischemic attack according to a clinical definition (i.e., new neurological symptoms or deficits lasting less than 24 hours with no new infarction on neuroimaging) ${ }^{21}$ as well as (iv) recurrent CAD during the follow-up period of 90 \pm 30 days since treatment onset. Safety was monitored by the recording of adverse and serious adverse events. Further, as specified within the trial data safety monitoring board (DSMB) charter, the DSMB assessed patient safety after enrolment of $80 \%$ of the original target sample size. The DSMB concluded that there were no safety issues at that time point and that the enrolment could be completed as planned. 


\section{Statistical analysis}

The sample size of the trial was calculated to be able to show the non-inferiority of aspirin to VKA treatment in CAD patients with regard to the primary endpoint in the per protocol population. We expected a primary endpoint rate of $7 \%$ in both treatment arms $(2 \%$ clinical outcomes $8,10,11,5 \%$ subclinical MRI-outcomes ${ }^{23}$ ). Thus, 169 evaluable participants for per protocol population analysis were required to ensure at least $80 \%$ power $(1-$ beta $=0.8)$ at a significance level of alpha $=5 \%$ (two-sided). In order to ensure that we would achieve the number of 169 required patients in the per protocol population we performed a pre-planned (protocol version 3.1, dated 27.6.2016), blinded interim analysis after recruitment of $80 \%$ (i.e. $n=136$ ) of the initial target sample size. We neither analysed treatment allocations nor the occurrence of primary outcomes. Instead, we inspected the number of patients with major protocol deviations, who would not meet the criteria for per-protocol analysis. These were 18 among 136 recruited patients, amounting to a rate of $13.2 \%$ patients with major protocol violations. By extrapolating this rate, we estimated that a total of 194 participants had to be recruited to have data of at least 169 evaluable patients in the per protocol population.

Continuous data are presented as arithmetic mean and standard deviation or median and interquartile range as appropriate. For categorical variables, absolute and relative frequencies are presented. To address the main objective, the absolute risk difference between patients treated with aspirin and VKA is presented together with its $95 \%$ confidence interval using the Wilson's method (continuity-corrected modification of the Wilson's score method Newcombe, 1998). In addition, a test for the hull-hypothesis (absolute risk difference=12\%) was estimated using the $\mathrm{Z}_{\mathrm{cu}}$ statistics as suggested by Kawasaki et al.. ${ }^{24}$ 
Non-inferiority would be declared if the upper limit of the two-sided $95 \%$ confidence interval (CI) of the absolute risk difference is smaller than $12 \%$ (non-inferiority margin).

Due to the absence of unbiased information about treatment effects when TREAT-CAD was designed, the margin could not be based on statistically guided estimates. Thus, the margin reflected the absolute difference of events, for which clinicians might still accept non-inferiority between both treatment arms, although this decision is debatable. This rather large margin was defined considering, that the primary endpoint included clinical as well as subclinical MRIoutcomes.

The main analysis was performed on the per protocol population dataset. As sensitivity analyses, the primary analysis was repeated on the (i) full analysis dataset with worst case and best case imputations for missing outcome data (intention-to-treat-principle) and inverse probability weighting in order to assess the impact of patients excluded from the per-protocol population, (ii) on clinical outcomes only, and (iii) on MRI-outcomes only. No multiplicity adjustments have been made. Analyses were conducted using the statistical software package R, Version 3.6.3.

\section{Role of the funding sources}

The funders of this study had no role in study design, data collection, data analysis, data interpretation, or writing of the report. All authors confirm that they had full access to all the data in the study and accept responsibility for the decision to submit for publication. 
Engelter, Traenka et al. TREAT-CAD main results - 17 -

\section{RESULTS}

\section{TRIAL PATIENTS}

From September 2013 through December 2018, we enrolled 194 patients in 10 centers. Mean age was 45.9 years (SD 11.0, range 22-70 years) and 123 (63.4\%) patients were male. 129/194 patients $(66.5 \%)$ presented with ischemic events. Purely local symptoms were reported in 65/194 (33.5\%) patients. $102(52.6 \%)$ patients had ischemic MRI-lesions at baseline. All but 5 patients had had clinical ischemic events. 100 patients were randomly assigned to receive aspirin and 94 to receive VKA (phenprocoumon $n=78$, acenocoumarol $n=12$, and warfarin $n=4$ ). Fifty-one patients received heparin followed by VKA, while 43 received VKA alone. Mean follow-up time was 90.8 days (range 83-104 days) in the aspirin group and 90.5 days (range 87-104 days) in the VKA group, respectively.

Twenty-one (10.8\%) patients did not meet the criteria for per-protocol analysis for reasons outlined in the study flow chart (figure 1). This included all 14 patients with cross-over to the other treatment arm. Although all patients had brain and cervical MRI scans at baseline, these were incomplete in one patient treated with aspirin and one treated with VKA, respectively.

The per protocol population consisted of 173 patients ( $89.2 \%$ of all study patients), of whom 91 received aspirin and 82 received VKA. Among per-protocol patients, CAD involved the carotid artery in 115 patients, and the vertebral artery in 61 patients including 3 patients with involvement of the carotid plus the vertebral artery. Mural hematoma was the most frequent imaging characteristic identified in baseline MRI in 165 (95.4\%) patients.

Prior to study entry, acute revascularization procedures were performed in 23 patients (13.3\%) of the per protocol population overall, and in $25.5 \%$ of those patients presenting with stroke (23/90). In these patients, enrolment was allowed only after a 24-hour interval as specified in the inclusion 
Engelter, Traenka et al. TREAT-CAD main results - 18 -

criteria. Study treatment was started at a median of three days after hospital admission, without any difference between both treatment arms. Detailed history taking revealed that the very first CADsymptom - pain in most patients - had occurred at a median of seven days prior to start of study medication.

Baseline characteristics are summarized in table 1 and were comparable in both groups.

\section{MAIN ANALYSES}

The primary endpoint occurred in 33 patients amounting to an overall event rate of $19.1 \%$ in the per protocol population. It was recorded in 21 of 91 patients $(23.1 \%)$ in the aspirin-group versus 12 of 82 patients $(14.6 \%)$ in the VKA group. The observed difference of the primary endpoint rate between groups was $8.5 \%$ with a $95 \%$ CI ranging from -4.3 to 21.2 . As the upper end of the $95 \%$ CI exceeded the predefined margin of $12 \%$, non-inferiority could not be declared ( $\mathrm{p}$ for noninferiority: 0.55). With the lower end of the $95 \%$ CI crossing zero, this result cannot be considered as proof of inferiority.

During follow-up, ischemic stroke occurred in seven (7.7\%) patients in the aspirin group (six brain infarcts, one retinal infarct, all ipsilateral to the CAD at baseline). These events occurred either on day one (five patients) or day seven (two patients). Five of these seven patients in the aspirin group had had ischemic strokes (or a retinal infarct) as presenting symptom with a mean NIHSS of 1.6 [range 0-4] points. Ischemic lesions at baseline were present in 5 and absent in 2 of these 7 patients. There were no ischemic strokes in the VKA group. The only major haemorrhage (upper gastrointestinal bleed) occurred in the VKA group (1.2\%) on day seven. There were no deaths. MRI-outcomes were recorded in 20 patients (22.0\%) treated with aspirin and in 11 patients $(13.4 \%)$ treated with VKA. The distribution of subtypes (i.e. ischemic and/or haemorrhagic) of MRIoutcomes are presented in table 2. MRI-outcomes were associated with clinical symptoms in six 
of $20(30 \%)$ patients in the aspirin group while none of the 11 patients in the VKA group with such MRI-outcomes showed clinical symptoms.

Of the 33 patients with primary endpoints, 32 presented with either clinical ischemic events, MRIlesions, or both at baseline.

There were 19 adverse events in the aspirin group, of which one was a serious adverse event (SAE) and 26 adverse events in the VKA group, of which six were SAEs (table 3). All seven patients recovered completely from the SAEs.

\section{SENSITIVITY ANALYSES}

Point estimates and 95\%-CIs of the event rates for (i) the full analysis set population, (ii) MRIoutcomes only, and (iii) clinical outcomes only in the per protocol population closely resemble those of the main analysis (figure 2).

Post-hoc-sensitivity analyses: First, in order evaluate whether crossovers to the other treatment arm had any impact on our key finding, we performed a post-hoc analysis "as treated". This analysis included all patients of the per protocol population plus the crossover patients; the latter included all patients who had been excluded from the per protocol population because they had not received the medication they were randomized to but that of the other treatment group (i.e. $n=9$ crossovers from VKA to aspirin and $n=2$ crossovers from aspirin to VKA). We did not include another 3 patients, because diagnosis of dissection had not been confirmed by central MR reading $(n=2)$ and because the other treatment had eventually not been started $(n=1)$. For patients analyzed "as treated", the observed difference of the primary endpoint rate between groups was $6.7 \%$ with a 95\% CI ranging from -5.3 to 18.8 , which was consistent to the main analysis.

Secondly, as the meaning of new subclinical haemorrhagic brain lesions as seen on paramagnetic (SWI, GRE) MRI sequences is not well established, we performed a post-hoc sensitivity analysis, 
Engelter, Traenka et al. TREAT-CAD main results - 20 -

excluding these lesions from the primary outcome. The observed difference between groups would have been $3.4 \%$ with a $95 \%$ CI ranging from -7.2 to 14.1 , which were concordant to the main analysis.

Thirdly, the - numerically - higher rate of acute recanalization procedures in the aspirin group may be a confounder, as acute recanalization procedures involve the risk of clinical or subclinical MRoutcomes as complication. We tried to minimize the influence of these procedures by requiring a 24-hour-delay before recruitment was allowed. However, periprocedural, clinical or subclinical outcomes occurring beyond the 24-hour-time interval, could have interfered. Among the 23 patients who had acute revascularization procedures before study participation, none had a clinical outcome event. However, seven patients had subclinical MR-lesions. These occurred in five of 15 patients in the aspirin-group and in two of the eight patients in the VKA-group who had prior revascularization procedures. Thus, we performed an additional post-hoc sensitivity analysis on the primary outcome excluding all patients who had had acute recanalization procedure prior to enrolment in the trial.

Among those patients, the observed difference of the primary endpoint rate between groups was $4.9 \%$ with a $95 \%$ CI ranging from -7.7 to 17.6 and supported our main conclusion.

\section{DISCUSSION}

TREAT-CAD is a randomised controlled trial comparing aspirin versus VKA in cervical artery dissection using a composite endpoint of clinical and MRI-outcomes. The key finding is, that noninferiority of aspirin compared to VKA was not established.

Ischemic stroke, major bleeds, or their MRI surrogates occurred in every fourth to fifth patient treated with aspirin $(23.1 \%)$ but in only every seventh patient in the VKA group (14.6\%). As the 
upper end of the $95 \%$-CI of the resulting $8 \%$ absolute difference (i.e. $21 \%$ ) exceeded the predefined margin of $12 \%$, non-inferiority of aspirin compared to VKA was not established.

The finding was confirmed in sensitivity analyses across all study patients. Thus, the pre-defined methodological decision to base the main analysis on the per-protocol population had no influence on the key findings of the trial. More importantly, the analyses on between group differences in pure clinical outcomes or pure MRI-outcomes revealed the same results as the main analyses using the composite endpoint. If we had restricted the study to clinical outcomes only, aspirin would still not be shown to be non-inferior. The concordance across all components of the composite primary endpoints and across all sensitivity analysis are arguments against the randomness of our key finding.

Among the primary endpoints, both clinical and MRI-outcomes occurred numerically more often in the aspirin-group than in the VKA-group. For clinical outcomes, five of six meta-analyses across observational studies had suggested that ischemic strokes could be expected in similar frequency in both treatment groups. ${ }^{8-12}$, while one meta-analysis reported on a higher rate of ischemic strokes among patients treated with antiplatelets (6.9\%) as compared with patients who received anticoagulants $(2.3 \%){ }^{13}$

In TREAT-CAD, all seven ischemic strokes occurred in the aspirin group, while the only major haemorrhage occurred in the anticoagulation group. In comparison, in the CADISS-trial, ischemic strokes occurred - numerically - also more often in the antiplatelet group (i.e., three out of 124 patients) than in the VKA group (one out of 126) and the only major haemorrhage occurred in the VKA group as well. ${ }^{14}$ A simple study level meta-analysis across clinical outcomes (stroke, major haemorrhage, death) in both CADISS and TREAT-CAD showed no statistically significant 
Engelter, Traenka et al. TREAT-CAD main results - 22 -

difference between treatment groups (Aspirin 5.2\% vs. VKA 1.7\%, absolute difference $=3.5 \%$, CI $=[-0.7 \%, 7.7 \%], \mathrm{p}=0.12)$.

Reasons for the higher rate of ischemic strokes in the aspirin group in TREAT-CAD compared to CADISS remain elusive. Comparing the baseline characteristics of the aspirin group of TREATCAD with that of CADISS, showed no signal that the aspirin group in TREAT-CAD comprised patients with a particularly high risk for subsequent stroke. In CADISS, $1 / 4$ of the patients in the antiplatelet group received aspirin plus clopidogrel. In TREAT-CAD, all patients received aspirin as a monotherapy, albeit in a higher daily dose of 300mg. Whether this variation could convincingly explain the difference in clinical event rates remains unclear.

In CAD, most strokes reportedly occur early after initial symptoms. ${ }^{25}$ However, in TREAT-CAD, time from symptom onset to treatment start did not differ between both treatment groups and is therefore not a plausible reason for the higher stroke rate in the aspirin group. Previous research showed that occlusion of the dissected artery, multivessel dissection and vertebral artery as site of dissection may increase the risk factor for delayed stroke (ie, stroke after hospital admission) in CAD patients. ${ }^{26}$ Significant differences in baseline characteristics between groups were absent. Still, occlusion of the dissected artery and multivessel dissections were - numerically - more frequent in the aspirin group than in the VKA group (35\% vs $28 \%$ and $9 \%$ vs $6 \%$ ). In turn, the vertebral artery as site of dissection was more common in the VKA (42\%) group than in the aspirin group (30\%). Likewise, acute ischemic lesion present at baseline imaging as a determinant for recurrent lesions ${ }^{23}$ was - numerically - more frequent in the VKA-group (57\%) than in the aspiringroup (52\%). Moreover, ischemic stroke as presenting symptom, which is a known strong predictor for recurrent stroke in $\mathrm{CAD}$ patients was present as often in the aspirin-group (51.6\%) as in the 
VKA-group (52.4\%). Thus, the observed between group differences in event rates characteristics may not be attributable to differences in baseline characteristics.

The consideration, that those patients in the VKA arm, who had received the recommended bridging with heparin or low molecular weight heparin in addition to VKA before reaching the anticoagulation target, might have had a better protection against early ischemic strokes - in particular on day one - than aspirin-treated patients, might serve as a possible explanation for the observed rate of ischemic strokes higher in the aspirin than in the VKA group.

Like for ischemic strokes as clinical outcome, numerically more (i.e. 11 versus seven) subclinical ischemic MRI-lesions occurred in the aspirin group than in the VKA-group. This imbalance contrasts with prior, non-randomised observations where the type of antithrombotic treatment had no impact on the occurrence of new ischemic lesions in the observational cohort (i.e. OR 1.0 (95\% CI $[0.32-3.15]) .^{23}$

Counterintuitively, numerically more haemorrhagic MRI-lesions occurred in the aspirin group $(n=11)$ than in the anticoagulation group $(n=5)$. However, the interpretation of the pathophysiological meaning of small paramagnetic MRI-lesions may be more complex, as some of these lesions may reflect haemorrhagic transformation ${ }^{27}$, which occurs also in the context of non-CAD ischemic strokes ${ }^{28}$ and can be accompanied by ischemic brain lesions, as was the case in some of our patients.

With one exception, all primary endpoints - clinical or MRI-outcomes - occurred in those patients who had either ischemic events or MRI-lesions at baseline, underlining the prognostic importance of these characteristics as suggested by prior research. ${ }^{14,}{ }^{26}$ However, TREAT-CAD was not powered to perform conclusive analyses in subgroups. 
MRI-outcomes occurred more often (i.e. about 4 times) than clinical outcome events, which was in line with our assumptions when designing the trial. More importantly, the consistent findings of clinical and MRI-outcomes strengthens the conclusion of the trial.

The clinical meaning of MRI-outcomes without accompanying clinical symptoms is debated and indeed unclear. ${ }^{29}$ While some studies have suggested, that MRI outcomes may be useful as surrogates for clinical outcomes ${ }^{17,18,30}$ MRI outcomes cannot entirely replace clinical endpoints. Nevertheless, it is possible that MRI-outcomes are associated with the occurrence of psychosocial sequela, which have been reported in a high rate in otherwise well-recovered CAD-patients. ${ }^{31}$ As several CAD-patients are in their 30s-50s lacking relevant comorbidities, we assume that the occurrence of new ischemic or haemorrhagic brain lesions is of disadvantage, no matter if they are reflected in neurological exams, or not.

An important limitation involves the rather large non-inferiority margin, which - due to the absence of reliable data at the time of the design of the trial - was not guided by calculated estimates, but reflected the limit for non-inferiority between both treatment arms as chosen when TREAT-CAD was designed. This decision is debatable. The larger the margin, the more a higher number of events in the aspirin group would still be considered non-inferior. Thus, the rather large margin would have been a more important limitation, if we had shown non-inferiority than in the current situation where non-inferiority has not been shown - despite a large margin.

Furthermore, the large 95\%-CI for the primary endpoint showed the limited precision for the observed difference in the treatment effect between both arms and urge to a cautious interpretation of the point estimate. In addition, the use of a composite primary outcome, although used in a prior 
$\mathrm{RCT}^{19}$, must not support the idea that all components are equally important and that they have the same treatment response.

A major limitation of the chosen non-inferiority design is, that TREAT-CAD could not prove that aspirin is worse than VKA in CAD, as the study was not designed and powered to address the question of superiority of either treatment. It is important to emphasize, that the fact that aspirin was not shown non-inferior to VKA, does not mean that aspirin is worse and VKA is superior to aspirin. Moreover, as most study patients presented with minor-to moderate strokes, TIAs or nonischemic symptoms, the applicability of our key findings to patients with major or disabling stroke is less clear.

As strengths, all TREAT-CAD centers had a special interest in CAD diagnosis and treatment. Their expertise translated into a rate of verified dissections of $>97 \%$, solving the problem that the correct diagnosis of CAD in the acute setting is challenging. ${ }^{15}$ Independent and central adjudication of both clinical and imaging outcomes support the validity of the results. The fact that clinical and MRI-outcomes were concordant are arguments against spurious findings.

In TREAT-CAD we did not use dual antiplatelet therapy, as this acute stroke treatment regimen was not well established when the study was designed. Given the results of the CHANCE-trial ${ }^{32}$ and the POINT-trial ${ }^{33}$, the use of dual antiplatelets might be considered in future CAD-trials as well, as this has not been specifically tested neither in CADISS nor TREAT-CAD. Likewise, a shorter than three months treatment duration could be tested, given the early occurrence of ischemic events observed in TREAT-CAD.

Further, in TREAT-CAD we were not allowed to include direct oral anticoagulants in the anticoagulation arm for regulatory reasons. Recently, in small case series, direct oral anticoagulants had been used in CAD-patients. ${ }^{34-37}$ In general, direct oral anticoagulants have a more favourable 
benefit-risk ratio and are more conveniently applicable than VKAs. However, it is unclear whether these advantages include patients with CAD. Thus, benefits and harms of direct oral anticoagulants in CAD should be compared to VKA in future trials applying a design similar to TREAT-CAD.

In conclusion, in patients with cervical artery dissection, aspirin was not non-inferior to VKA.

Data sharing: upon reasonable request to the corresponding author, trial data can be made available. Such requests must be accompanied by detailed study proposals, a description of study objectives as well as a statistical analysis plan. Additional material might by requested during the assessments of the study eligibility for data sharing, which must be approved by the corresponding author (i.e. Sponsor-Investigator of the trial), the trial steering committee and principal investigators of each centre. Each request will be checked for compatibility with regulatory (ethics committees) requirements as well as compatibility with patient informed consent.

\section{References}

1. Debette $S$ and Leys D. Cervical-artery dissections: predisposing factors, diagnosis, and outcome. Lancet neurology. 2009; 8: 668-78.

2. Engelter ST, Traenka C and Lyrer P. Dissection of Cervical and Cerebral Arteries. Curr Neurol Neurosci Rep. 2017; 17: 59.

3. Menon RK, Markus HS and Norris JW. Results of a UK questionnaire of diagnosis and treatment in cervical artery dissection. Journal of neurology, neurosurgery, and psychiatry. 2008; 79: 612.

4. Ahmed N, Steiner T, Caso V, Wahlgren N and participants E-Ks. Recommendations from the ESOKarolinska Stroke Update Conference, Stockholm 13-15 November 2016. Eur Stroke J. 2017; 2: 95-102.

5. Brott TG, Halperin JL, Abbara S, et al. 2011 ASA/ACCF/AHA/AANN/AANS/ACR/ASNR/CNS/SAIP/SCAI/SIR/SNIS/SVM/SVS guideline on the management of patients with extracranial carotid and vertebral artery disease: executive summary. Stroke; a journal of cerebral circulation. 2011; 42: e420-63.

6. Powers WJ, Rabinstein AA, Ackerson T, et al. Guidelines for the Early Management of Patients With Acute Ischemic Stroke: 2019 Update to the 2018 Guidelines for the Early Management of Acute Ischemic Stroke: A Guideline for Healthcare Professionals From the American Heart Association/American Stroke Association. Stroke; a journal of cerebral circulation. 2019; 50: e344-e418. 
7. Ringelstein EB, Dittrich $\mathrm{R}$ and al. e. S1-Leitlinie Spontane Dissektionen der extra- und intrakraniellen hirnversorgenden Arterien. 2016. Hrsg Leitlinien für Diagnostik und Therapie in der Neurologie. 2016.

8. Menon R, Kerry S, Norris JW and Markus HS. Treatment of cervical artery dissection: a systematic review and meta-analysis. Journal of neurology, neurosurgery, and psychiatry. 2008; 79: 1122-7.

9. Kennedy F, Lanfranconi S, Hicks $C$, et al. Antiplatelets vs anticoagulation for dissection: CADISS nonrandomized arm and meta-analysis. Neurology. 2012; 79: 686-9.

10. Lyrer $\mathrm{P}$ and Engelter $\mathrm{S}$. Antithrombotic drugs for carotid artery dissection. The Cochrane database of systematic reviews. 2010: CD000255.

11. Sarikaya H, da Costa BR, Baumgartner RW, et al. Antiplatelets versus anticoagulants for the treatment of cervical artery dissection: Bayesian meta-analysis. PloS one. 2013; 8: e72697.

12. Chowdhury MM, Sabbagh CN, Jackson D, Coughlin PA and Ghosh J. Antithrombotic Treatment for Acute Extracranial Carotid Artery Dissections: A Meta-Analysis. Eur J Vasc Endovasc Surg. 2015; 50: 14856.

13. Kim YK and Schulman S. Cervical artery dissection: pathology, epidemiology and management. Thromb Res. 2009; 123: 810-21.

14. Markus HS, Hayter E, Levi C, et al. Antiplatelet treatment compared with anticoagulation treatment for cervical artery dissection (CADISS): a randomised trial. Lancet neurology. 2015; 14: 361-7.

15. Kasner SE. CADISS: a feasibility trial that answered its question. Lancet neurology. 2015; 14: 3423.

16. Bonati LH, Jongen LM, Haller $\mathrm{S}$, et al. New ischaemic brain lesions on MRI after stenting or endarterectomy for symptomatic carotid stenosis: a substudy of the International Carotid Stenting Study (ICSS). Lancet neurology. 2010; 9: 353-62.

17. O'Donnell MJ, Eikelboom JW, Yusuf S, et al. Effect of apixaban on brain infarction and microbleeds: AVERROES-MRI assessment study. American heart journal. 2016; 178: 145-50.

18. Sharma M, Hart RG, Smith EE, et al. Rivaroxaban for Prevention of Covert Brain Infarcts and Cognitive Decline: The COMPASS MRI Substudy. Stroke; a journal of cerebral circulation. 2020; 51: 29019.

19. Sondergaard L, Kasner SE, Rhodes JF, et al. Patent Foramen Ovale Closure or Antiplatelet Therapy for Cryptogenic Stroke. The New England journal of medicine. 2017; 377: 1033-42.

20. Traenka C, Gensicke H, Schaedelin S, et al. Biomarkers and antithrombotic treatment in cervical artery dissection - Design of the TREAT-CAD randomised trial. European Stroke Journal. 2020.

21. Amarenco P, Lavallee PC, Monteiro Tavares L, et al. Five-Year Risk of Stroke after TIA or Minor Ischemic Stroke. The New England journal of medicine. 2018; 378: 2182-90.

22. Hayreh SS and Zimmerman MB. Central retinal artery occlusion: visual outcome. Am J Ophthalmol. 2005; 140: 376-91.

23. Gensicke H, Ahlhelm F, Jung $\mathrm{S}$, et al. New ischaemic brain lesions in cervical artery dissection stratified to antiplatelets or anticoagulants. European journal of neurology : the official journal of the European Federation of Neurological Societies. 2015; 22: 859-65, e61.

24. Kawasaki Y, Zhang Z and Miyaoka E. Comparisons of Test Statistics for Noninferiority Test for the Difference between Two Independent Binominal Proportions. American Journal of Biostatistics. 2010; 1: 23-31.

25. Morris NA, Merkler AE, Gialdini G and Kamel H. Timing of Incident Stroke Risk After Cervical Artery Dissection Presenting Without Ischemia. Stroke; a journal of cerebral circulation. 2017; 48: 551-5.

26. Lichy C, Metso A, Pezzini A, et al. Predictors of delayed stroke in patients with cervical artery dissection. International journal of stroke : official journal of the International Stroke Society. 2015; 10: 360-3. 
27. Mustanoja S, Haapaniemi E, Putaala J, Strbian D, Kaste M and Tatlisumak T. Haemorrhagic transformation of ischaemic stroke in young adults. International journal of stroke : official journal of the International Stroke Society. 2014; 9 Suppl A100: 85-92.

28. Greenberg SM, Vernooij MW, Cordonnier C, et al. Cerebral microbleeds: a guide to detection and interpretation. Lancet neurology. 2009; 8: 165-74.

29. Bae HJ and Debette S. Commentary on the Cervical Artery Dissection in Stroke Study Trial. Stroke; a journal of cerebral circulation. 2016; 47: 1413-5.

30. Traenka C, Engelter ST, Brown MM, Dobson J, Frost C and Bonati LH. Silent brain infarcts on diffusion-weighted imaging after carotid revascularisation: A surrogate outcome measure for procedural stroke? A systematic review and meta-analysis. Eur Stroke J. 2019; 4: 127-43.

31. Speck V, Noble A, Kollmar R and Schenk T. Diagnosis of spontaneous cervical artery dissection may be associated with increased prevalence of posttraumatic stress disorder. Journal of stroke and cerebrovascular diseases : the official journal of National Stroke Association. 2014; 23: 335-42.

32. Wang $Y$, Wang $Y$, Zhao $X$, et al. Clopidogrel with aspirin in acute minor stroke or transient ischemic attack. The New England journal of medicine. 2013; 369: 11-9.

33. Johnston SC, Easton JD, Farrant M, et al. Clopidogrel and Aspirin in Acute Ischemic Stroke and HighRisk TIA. The New England journal of medicine. 2018; 379: 215-25.

34. Mustanoja S, Metso TM, Putaala J, et al. Helsinki experience on nonvitamin $\mathrm{K}$ oral anticoagulants for treating cervical artery dissection. Brain Behav. 2015; 5: e00349.

35. Cappellari M and Bovi P. Direct oral anticoagulants in patients with cervical artery dissection and cerebral venous thrombosis. A case series and review of the literature. Int J Cardiol. 2017; 244: 282-4.

36. Malferrari G, Laterza D, Valzania F, Monaco D, Silingardi M and Pizzini AM. Rivaroxaban in cervical and "cervico-cerebral" artery dissections: a new therapeutic option? Neurol Sci. 2019; 40: 1591-6.

37. Caprio FZ, Bernstein RA, Alberts MJ, et al. Efficacy and safety of novel oral anticoagulants in patients with cervical artery dissections. Cerebrovasc Dis. 2014; 38: 247-53. 
Table 1: Characteristics of Patients at Baseline

\begin{tabular}{|c|c|c|c|c|}
\hline \multirow[t]{2}{*}{ Characteristic } & \multicolumn{2}{|c|}{ Per protocol population (n=173) } & \multicolumn{2}{|c|}{ Full analysis set population $(n=194)$} \\
\hline & Aspirin $(n=91)$ & VKA $(n=82)$ & Aspirin $(n=100)$ & VKA $(n=94)$ \\
\hline Age (years, mean (SD)) & $46.7(10.2)$ & $45.5(11.6)$ & $46.6(10.6)$ & $45.5(11.6)$ \\
\hline Male sex, n (\%) & $56(61.5)$ & $54(65.9)$ & $62(62.0)$ & $61(64.9)$ \\
\hline \multicolumn{5}{|l|}{ Site of dissection } \\
\hline Internal Carotid Artery, $\mathrm{n}(\%)$ & $65(71.4)$ & $50(61.0)$ & $72(72.0)$ & $58(61.7)$ \\
\hline Vertebral Artery, n (\%) & $27(29.7)$ & $34(41.5)$ & $29(29.0)$ & $38(40.4)$ \\
\hline Multivessel dissection, $\mathrm{n}(\%)$ & $8(8.8)$ & $5(6.1)$ & $9(9.0)$ & $5(5.3)$ \\
\hline Occlusion of dissected artery, $n(\%)$ & $32 / 90(35.2)$ & $23(28.0)$ & 35/99 (35.4) & $26(27.7)$ \\
\hline Mural hematoma of dissected artery at baseline, $n(\%)$ & $89(97.8)$ & $76(92.7)$ & $97(97.0)$ & $88(93.6)$ \\
\hline \multicolumn{5}{|l|}{ Presenting signs and symptoms } \\
\hline \multicolumn{5}{|l|}{ Cerebral Ischemic events } \\
\hline Ischemic stroke & $47(51.6)$ & $43(52.4)$ & $52(52.0)$ & $49(52.1)$ \\
\hline Transient ischemic attack & $12(13.2)$ & $10(12.2)$ & $14(14.0)$ & $10(10.6)$ \\
\hline Retinal infarct & $3(3.3)$ & $1(1.2)$ & $3(3.0)$ & $1(1.1)$ \\
\hline Amaurosis fugax & $2(2.2)$ & $5(6.1)$ & $2(2.0)$ & $7(7.4)$ \\
\hline \multicolumn{5}{|l|}{ Local signs } \\
\hline Cervical pain & $46(50.5)$ & $41(50.0)$ & $51(51.0)$ & $47(50.0)$ \\
\hline Headache & $65(71.4)$ & $54(65.9)$ & $72(72.0)$ & $64(68.1)$ \\
\hline Cranial nerve palsy & $11(12.1)$ & $7(8.5)$ & $11(11.0)$ & $7(7.4)$ \\
\hline Horner's syndrome & $32(35.2)$ & $28(34.1)$ & $36(36.0)$ & $34(36.2)$ \\
\hline Tinnitus & $13(14.3)$ & $4(4.9)$ & $13(13.0)$ & $6(6.4)$ \\
\hline Modified Rankin Scale Score at baseline, mean (SD)** & $1.8(1.2)$ & $1.8(1.3)$ & $1.8(1.2)$ & $1.8(1.3)$ \\
\hline NIHSS Score at baseline, mean (SD)** & $2.1(2.9)$ & $2.5(4.3)$ & $2.1(2.7)$ & $2.5(4.1)$ \\
\hline $\begin{array}{l}\text { Time between hospital admission and treatment, days } \\
\text { (median),(IQR)) }\end{array}$ & $2.8(1.2,4.9)$ & $3.0(1.5,5.4)$ & $2.9(1.3,4.8)$ & $3.0(1.5,5.4)$ \\
\hline
\end{tabular}


Engelter, Traenka et al. TREAT-CAD main results - 30 -

\begin{tabular}{|c|c|c|c|c|}
\hline $\begin{array}{l}\text { Time between onset of very first symptom attributable to } \\
\text { CAD and treatment, days (median), (IQR)) }\end{array}$ & $7.0(4.0,10.0)$ & $6.0(3.2,8.8)$ & $7.0(4.0,10.0)$ & $6.0(4.0,9.0)$ \\
\hline \multicolumn{5}{|l|}{ Acute recanalization therapies } \\
\hline Intravenous thrombolysis & $12(13.2)$ & $7(8.5)$ & $13(13.0)$ & $9(9.6)$ \\
\hline Endovascular therapy / bridging & $3(3.3)$ & $1(1.2)$ & $3(3.0)$ & $2(2.1)$ \\
\hline \multicolumn{5}{|l|}{ Risk factors } \\
\hline Hypertension & $30(33.0)$ & $25(30.5)$ & $32(32.0)$ & $28(29.8)$ \\
\hline Hypercholesterolemia & $18(19.8)$ & $18(22.0)$ & $19(19.0)$ & $20(21.3)$ \\
\hline Diabetes & $1(1.1)$ & $3(3.7)$ & $1(1.0)$ & $3(3.2)$ \\
\hline History of smoking & $43(47.3)$ & $42(51.2)$ & $46(46.0)$ & $47(50.0)$ \\
\hline Migraine with aura & $13(14.3)$ & $10(12.2)$ & $13(13.0)$ & $11(11.7)$ \\
\hline Migraine without aura & $17(18.7)$ & $7(8.5)$ & $18(18.0)$ & $8(8.5)$ \\
\hline $\begin{array}{l}\text { Mechanical trigger event within } 4 \text { weeks prior to } \\
\text { enrolment }\end{array}$ & $12(13.2)$ & $16(19.5)$ & $13(13.0)$ & $18(19.1)$ \\
\hline Infection within 4 weeks prior to enrolment & $24(26.4)$ & $15(18.3)$ & $27(27.0)$ & $18(19.1)$ \\
\hline \multicolumn{5}{|l|}{ Baseline MR characteristics*** } \\
\hline Acute ischemic MR lesion at baseline & $47(51.6)$ & $47(57.3)$ & $51(51.5)$ & $51(54.8)$ \\
\hline Haemorrhagic MR lesion at baseline & $7(7.7)$ & $5(6.1)$ & $7(7.0)$ & $6(6.4)$ \\
\hline Verification of dissection in central reading & $91(100)$ & $82(100)$ & $98(98)$ & $92(97.9)$ \\
\hline
\end{tabular}

Legend: * $\mathrm{n}=11$ had multiple ischemic events, ** In patients with ischemic stroke. *** Full analysis set population: Baseline MRI incomplete in two patients (n=1 Aspirin group and $n=1$ VKA group). 
Data on primary endpoint available Pts with incomplete follow-up data

Aspirin $(\mathrm{n}=91) \quad$ VKA $(\mathrm{n}=82)$

91

0

no. of patients (\%)

82
Full analysis set population ( $p=194)$

Aspirin $(\mathrm{n}=100) \quad$ VKA $(\mathrm{n}=94)$

$\begin{array}{cc}97 & 93 \\ 3 & 1\end{array}$

no. of patients $(\%)$

\section{Primary endpoint, total}

$21(23.1)$

12 (14.6)

22/97 (22.7)

$12 / 93(12.9)$

\section{Components of the composite primary}

\section{endpoint}

\section{Clinical outcomes}

Ischemic stroke*

7 (7.7)

$1(1.2)$

$7(7.2)$

$1(1.1)$

Haemorrhage

Major extracranial haemorrhage

$7(7.7)$

$0(0.0)$

$7(7.2)$

$0(0.0)$

Symptomatic intracranial

$0(0.0)$

$1(1.2)$ haemorrhage

Death

$0(0.0)$

$0(0.0)$

$0(0.0)$

$1(1.1)$

$0(0.0)$

$0(0.0)$

$0(0.0)$

$0(0.0)$

\section{MRI-outcomes (all)}

New acute ischemic brain lesions

New haemorrhagic brain lesion

New acute ischemic or new

$20(21.9)$

$9(9.9)$

$9(9.9)$

$11(13.4)$

$0(0.0)$

$0(0.0)$

haemorrhagic lesions

\section{MRI-outcomes without symptoms}

New acute ischemic brain lesions

New haemorrhagic brain lesion

Both, new acute ischemic plus new

2 (2.2)

(7.3)

$4(4.9)$

$1(1.2)$

$21(21.6)$

$10(10.3)$

$11(11.8)$

$9(9.3)$

(6.5)

$2(2.1)$

$4(4.3)$

$1(1.1)$

haemorrhagic lesions

\section{Secondary endpoints}

\begin{tabular}{|c|c|c|c|c|}
\hline Recurrent dissection & $3(33)$ & $2(24)$ & $3 / 07(31)$ & (2/03 (2) \\
\hline Increase of vessel wall hematoma & $1(1.1)$ & $1(1.2)$ & $1 / 97(1.0)$ & $5 / 93(5.4)$ \\
\hline Transient ischemic attack & $0(0.0)$ & $2(2.4)$ & $0 / 97(0.0)$ & $2 / 93(2.2)$ \\
\hline $\begin{array}{l}\text { Excellent functional outcome } \\
\text { (mRS } 0-1 \text { at } 3 \text { months) }\end{array}$ & $70(76.9)$ & $62 / 81(76.5)$ & $78 / 99(78.8)$ & $72 / 92(78.3)$ \\
\hline $\begin{array}{l}\text { Independence in activities of daily living } \\
\text { (mRS 0-2 at } 3 \text { months) }\end{array}$ & $88(96.7)$ & $80 / 81(98.8)$ & 96/99 (97) & $90 / 92(97.8)$ \\
\hline
\end{tabular}

Legend: * including one patient with retinal infarction.

$\begin{array}{cccc}14(15.4) & 11(13.4) & 15(15.5) & 11(11.8) \\ 3(3.3) & 6(7.3) & 4(4.1) & 6(6.5) \\ 9(9.9) & 4(4.9) & 9(9.3) & 4(4.3) \\ 2(2.2) & 1(1.2) & 2(2.1) & 1(1.1)\end{array}$


Table 3: Adverse Events

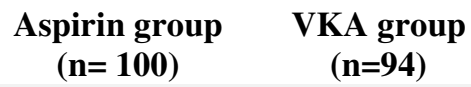

Implantation of a knee endoprosthesis

1

Hypertension

Syncope

Nausea

Gastroenteritis

Sore throat

Stomach pain

Xerostomia

Cough

Viral infection

Renal cystic tumor, incidental finding

Exercise-induced dyspnea

Depressive episode

Tachycardia

Suspicion of PEG-tube infection

Obstructive sleep apnea syndrome

Epistaxis

Constipation associated with opioid intake

Alopecia

Worsening of headache

Worsening of cervical pain

Accident induced hematoma on knee joint

Subconjunctival eye haemorrhage

Fall induced occipital laceration wound

Nephrolithiasis

Dizziness

Herpes Zoster

Benign paroxysmal positional vertigo

Panic attacks

Atrial fibrillation

Exanthema, possibly drug related

Loss of libido

Seizure

Migraine equivalent

Allergic reaction to MR contrast agent

Hospitalization due to suspected recurrent stroke

Total
1

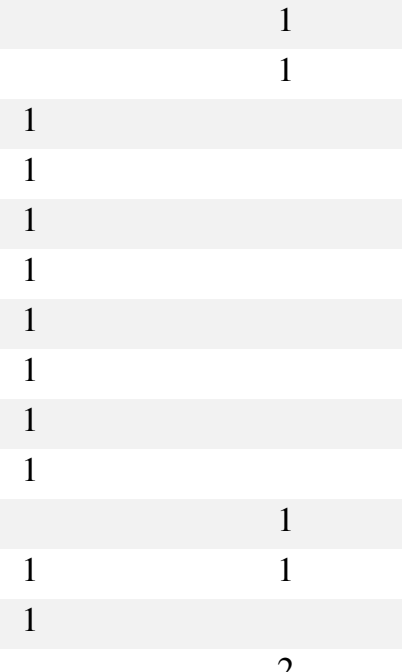

2

3

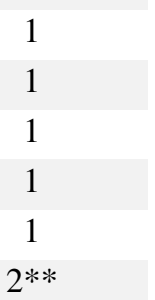

1

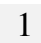

1

1

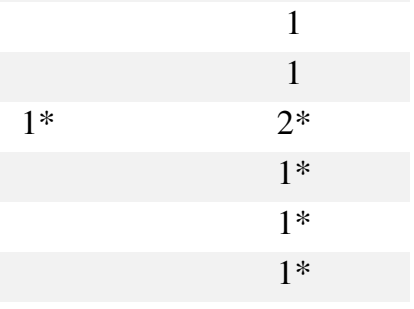

19

Legend: wording of adverse events as provided by center. In the aspirin group adverse events led to discontinuation $(n=1)$ or dose reduction $(n=1)$ of the allocated treatment in two patients. In the VKA group adverse events led to treatment crossovers to aspirin in three patients. * Events that fulfilled criteria of a Serious Adverse Event (SAE, e.g. leading to or prolongation of hospitalization, life-threatening). ** In one case fulfilled SAE criteria. 


\section{Figure 1: Study flow chart}

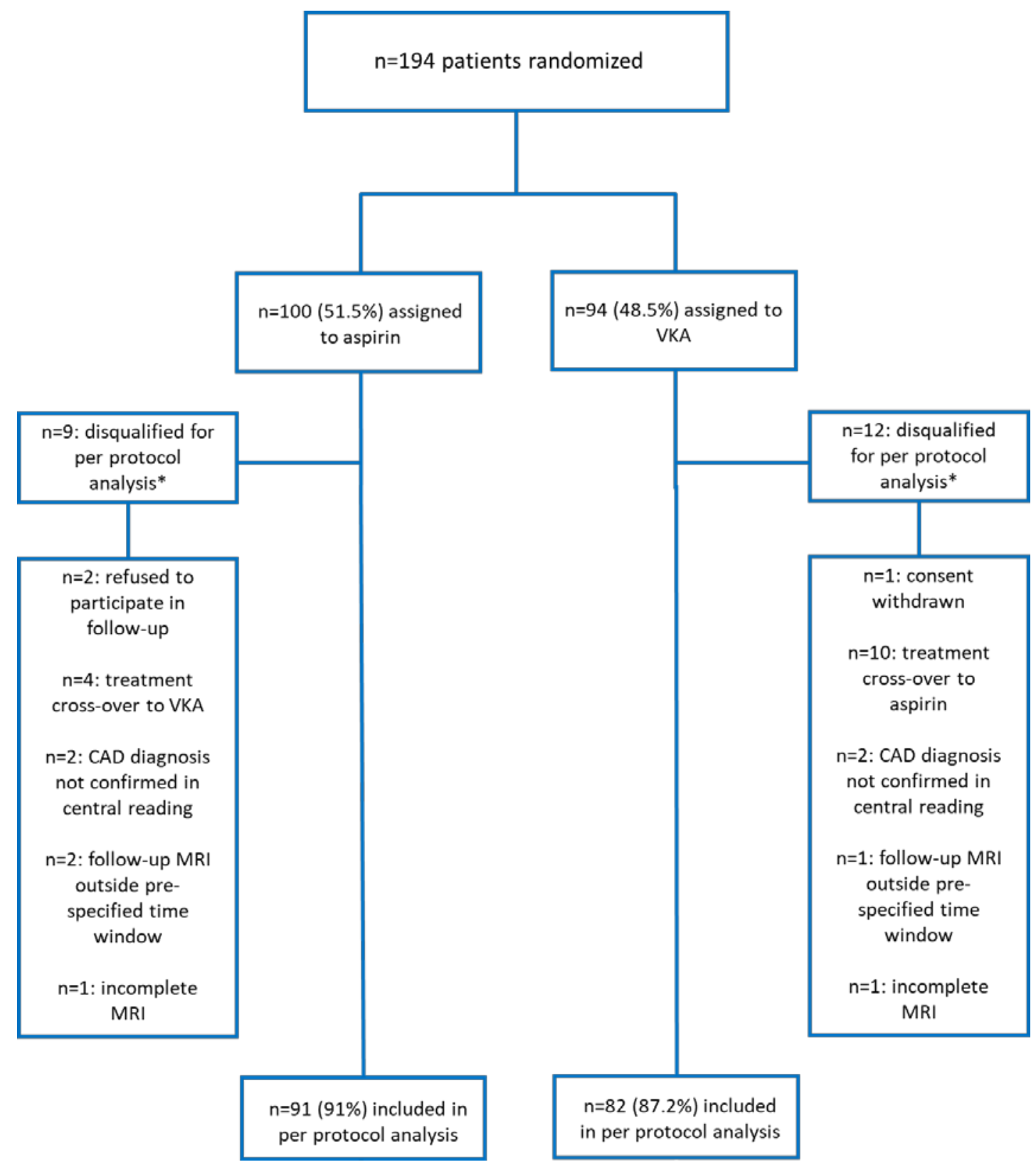

Legend: VKA refers to Vitamin K oral antagonists; CAD refers to cervical artery dissection. * Four patients had more than one of these criteria. 


\section{Figure 2. Forest plot of the main analysis and sensitivity analyses}

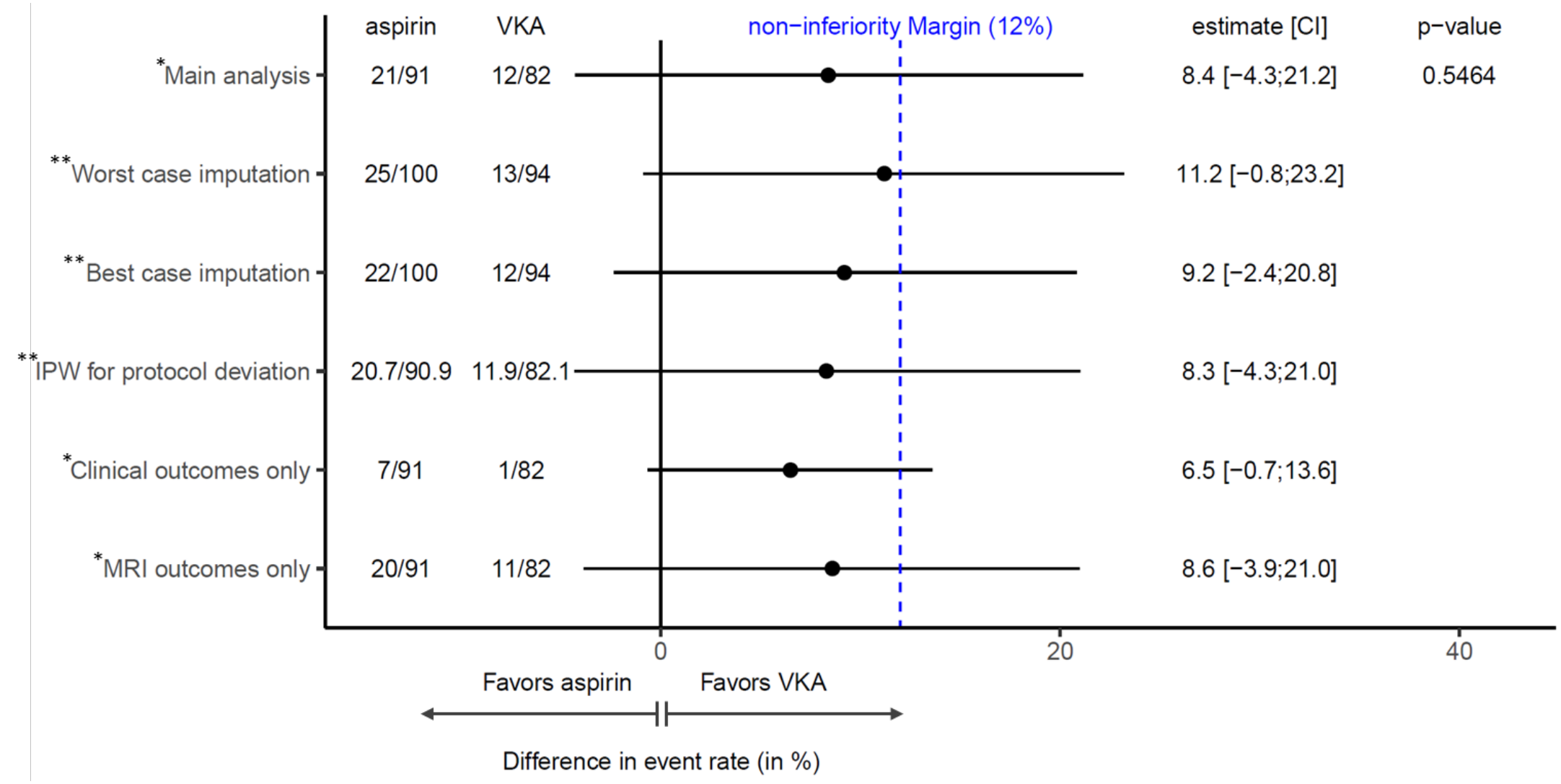

Legend: *Analyses were performed on the per protocol population dataset. **Analyses were performed on the full analysis set population dataset. VKA refers to Vitamin K oral antagonists. IPW refers to inverse probability weighting. Results are given as absolute risk differences (black dots) and the $95 \%$ confidence interval (black line) regarding the primary endpoint (i.e., the composite of clinical outcomes (i.e., stroke, major haemorrhage, death) and MRI-outcomes (i.e., new ischemic or haemorrhagic brain lesions). Non-inferiority of aspirin would be shown if the upper limit of the $95 \%$ confidence interval of the absolute risk difference between groups was less than $12 \%$ (non-inferiority margin). In the full analysis set population there was one additional MRI outcome (ischemic lesion) in a patient in the aspirin group. In this patient, CAD diagnosis was not confirmed in central reading. No further outcomes were detected in either group. In four (of 194) patients of the full analysis set population, information on presence or absence of primary endpoints was missing. Missing data were imputed. 